EPJ Web of Conferences 38, 14004 (2012)

DOI: $10.1051 /$ epjconf/20123814004

(C) Owned by the authors, published by EDP Sciences, 2012

\title{
Relativistic models for quasielastic electron and neutrino-nucleus scattering
}

\author{
Carlotta Giusti $^{1, \mathrm{a}}$ and Andrea Meucci ${ }^{1}$ \\ Dipartimento di Fisica, Università degli Studi di Pavia and \\ INFN, Sezione di Pavia, via Bassi 6 I-27100 Pavia, Italy
}

\begin{abstract}
Relativistic models developed within the framework of the impulse approximation for quasielastic (QE) electron scattering and successfully tested in comparison with electron-scattering data have been extended to neutrino-nucleus scattering. Different descriptions of final-state interactions (FSI) in the inclusive scattering are compared. In the relativistic Green's function (RGF) model FSI are described consistently with the exclusive scattering using a complex optical potential. In the relativistic mean field (RMF) model FSI are described by the same RMF potential which gives the bound states. The results of the models are compared for electron and neutrino scattering and, for neutrino scattering, with the recently measured charged-current QE (CCQE) MiniBooNE cross sections.
\end{abstract}

\section{Introduction}

The analysis of the recent neutrino-nucleus scattering cross sections measured by the MiniBooNE Collaboration [1,2] requires reliable theoretical models where all nuclear effects are well under control. The energy region explored requires a relativistic description of the process, where not only relativistic kinematics is considered but also nuclear dynamics and current operators are described within a relativistic framework. Within the QE kinematics domain, the treatment of the FSI between the ejected nucleon and the residual nucleus is an essential ingredient for the comparison with data.

Relativistic models for QE electron and neutrino-nucleus scattering, with different descriptions of FSI, are presented and compared in this contribution.

In spite of many similar aspects, electron and neutrinonucleus scattering are two different processes and it is not guaranteed that a model able to describe electron scattering data will be able to describe neutrino scattering data with the same accuracy. The large amount of data available for electron scattering and all the theoretical and experimental work done over several decades, which provided a wealth of detailed information on nuclear structure and dynamics [3,4], make electron scattering the best available guide to determine the predictive power of a nuclear model. A good description of electron scattering data can therefore be considered a necessary prerequisite for any model aimed to describe neutrino-nucleus cross sections.

In the QE region the nuclear response is dominated by the mechanism of one-nucleon knockout, where the probe interacts with a quasifree nucleon which is emitted from the nucleus with a direct one-step mechanism and the remaining nucleons are spectators. In the exclusive $\left(e, e^{\prime} p\right)$ reaction the outgoing nucleon is detected in coincidence with the scattered electron, the residual nucleus is left in a specific discrete eigenstate, and the final state is com-

\footnotetext{
a e-mail: Carlotta.Giusti@pv.infn.it.
}

pletely specified. In the inclusive $\left(e, e^{\prime}\right)$ scattering only the scattered electron is detected, the final nuclear state is not determined, and the cross section includes all the available final nuclear states. For an incident neutrino, neutralcurrent (NC) and charged-current (CC) scattering can be considered. In NC scattering only the emitted nucleon can be detected and the process is inclusive in the lepton sector (the final neutrino is not detected) and semi-inclusive in the hadron sector (the emitted nucleon is detected but the final nuclear state is not completely determined). In CC scattering the inclusive scattering where only the final lepton is detected can be treated with the same models used for the inclusive $\left(e, e^{\prime}\right)$ reaction.

For all these processes the cross section is obtained in the one-boson exchange approximation from the contraction between the lepton tensor, which, under the assumption of the plane-wave approximation for the initial and the final lepton wave functions, depends only on the lepton kinematics, and the hadron tensor, whose components are given by bilinear products of the matrix elements of the nuclear current between the initial and final nuclear states. Different but consistent models to calculate the hadron tensor in the exclusive and in the inclusive scattering have been developed and are outlined in the following sections.

\section{The exclusive $\left(e, e^{\prime} p\right)$ reaction}

The theoretical framework for the description of the exclusive $\left(e, e^{\prime} p\right)$ reaction is the distorted-wave impulse approximation (DWIA). The model is based on the following assumptions $[3,4]$ :

i) An exclusive process is considered, where the residual nucleus is left in a discrete eigenstate $n$ of its Hamiltonian. ii) The final nuclear state is projected onto the channel subspace spanned by the vectors corresponding to a nucleon, at a given position, and the residual nucleus in the state $n$. iii) The (one-body) nuclear-current operator does not connect different channel subspaces and also the initial state 
is projected onto the selected channel subspace. This is the assumption of the direct-knockout mechanism and of the IA.

The matrix elements of the nuclear current are then obtained in a one-body representation as

$$
\lambda_{n}^{1 / 2}\left\langle\chi^{(-)}\left|j^{\mu}(\boldsymbol{q})\right| \varphi_{n}\right\rangle
$$

where $j^{\mu}$ the one-body nuclear current, $\chi^{(-)}$is the singleparticle (s.p.) scattering state of the emitted nucleon, $\varphi_{n}$ the overlap between the ground state of the target and the final state $n$, i.e., a s.p. bound state, and the spectroscopic factor $\lambda_{n}$ is the norm of the overlap function, that gives the probability of removing a nucleon from the target leaving the residual nucleus in the state $n$. The s.p. bound and scattering states are eigenfunctions of a non Hermitian energydependent Feshbach-type optical potential and of its Hermitian conjugate at different energies. In standard DWIA calculations phenomenological ingredients are usually employed: the scattering states are eigenfunctions of a phenomenological optical potential determined through a fit to elastic nucleon-nucleus scattering data and the s.p. bound states are usually obtained from mean-field potentials.

The model can be formulated in a similar way within nonrelativistic DWIA [5] and relativistic RDWIA frameworks [6]. In RDWIA, calculations are performed with a relativistic nuclear-current operator and four-vector relativistic wave functions for the s.p. bound and scattering states. Both the DWIA and the RDWIA have been quite successful in describing $\left(e, e^{\prime} p\right)$ data in a wide range of nuclei and in different kinematics $[4,6-8]$.

\section{Inclusive lepton-nucleus scattering}

In the inclusive scattering only the outgoing lepton is detected, the final nuclear state is not determined, and all elastic and inelastic channels contribute. This requires a different treatment of FSI with respect to the exclusive process, where FSI are described by a complex optical potential whose imaginary part gives an absorption that reduces the calculated cross sections. The imaginary part accounts for the fact that in the elastic scattering, if other channels are open besides the elastic one, part of the incident flux is lost in the elastically scattered beam and goes to the other (inelastic) channels which are open. In the exclusive scattering only the channel $n$ is considered and it is correct to account for the flux lost in the considered channel. In the inclusive scattering all the channels contribute, the flux lost in a channel must be recovered in the other channels, and in the sum over all the channels the flux can be redistributed but must be conserved. If the inclusive cross section is obtained from the sum of all the integrated exclusive one-nucleon knockout processes, due to the interaction of the probe with all the individual nucleons of the nucleus, and FSI are described by a complex optical potential with an absorptive imaginary part, the flux is not conserved. The use of a complex optical potential seemed inconsistent with the requirement of flux conservation and in many calculations based on the relativistic IA real potentials have been adopted to describe FSI.

In the simplest approach FSI are simply neglected and the plane-wave approximation is assumed for the scattering wave functions (RPWIA). In a different approach an optical potential is used but only its real part is retained (rROP). The rROP conserves the flux but, independently of its numerical results, it is conceptually wrong because the optical potential has to be complex owing to the presence of open inelastic channels. The energy dependence of the optical potential reflects the different contributions of the inelastic channels which are open at each energy and, under such conditions, dispersion relations dictate that the optical potential must have a non vanishing imaginary part. In a different approach the scattering states are given by the same relativistic mean field potential considered in describing the initial nucleon states (RMF) $[9,10]$. The RMF model gives a consistent description of bound and scattering states, it fulfills the dispersion relations [11] and also the continuity equation.

In the Green's function (GF) model [12-18] FSI are described in the inclusive scattering by the same complex optical potential as in the exclusive scattering, but the imaginary part is used in the two cases in a different way and in the inclusive process it redistributes the flux in all the channels and the total flux is conserved.

In the GF model, under suitable approximations, which are basically related to the IA, the components of the hadron tensor are written in terms of the s.p. optical model Green's function. This result has been derived by arguments based on the multiple scattering theory [11] or by means of projection operators techniques within nonrelativistic [12] and relativistic [13-15] frameworks. The explicit calculation of the s.p. Green's function can be avoided [12-14] by its spectral representation, which is based on a biorthogonal expansion in terms of a non Hermitian optical-model Hamiltonian $\mathcal{H}$ and of its Hermitian conjugate $\mathcal{H}^{\dagger}$. The components of the hadron tensor are then obtained in the form [13]

$$
\begin{array}{r}
W^{\mu \mu}(q, \omega)=\sum_{n} \lambda_{n}\left[\operatorname{Re} T_{n}^{\mu \mu}\left(E_{\mathrm{f}}-\varepsilon_{n}, E_{\mathrm{f}}-\varepsilon_{n}\right)\right. \\
\left.-\frac{1}{\pi} \mathcal{P} \int_{M}^{\infty} \mathrm{d} \mathcal{E} \frac{1}{E_{\mathrm{f}}-\varepsilon_{n}-\mathcal{E}} \operatorname{Im} T_{n}^{\mu \mu}\left(\mathcal{E}, E_{\mathrm{f}}-\varepsilon_{n}\right)\right],
\end{array}
$$

where $E_{\mathrm{f}}$ denotes the energy of the final nuclear state, $\varepsilon_{n}$ denotes the eigenvalue of the discrete eigenstate $n$ of the residual nucleus, and

$$
\begin{aligned}
T_{n}^{\mu \mu}(\mathcal{E}, E) & =\left\langle\varphi_{n}\left|\hat{j}^{\mu \dagger}(\boldsymbol{q}) \sqrt{1-\mathcal{V}^{\prime}(E)}\right| \tilde{\chi}_{\mathcal{E}}^{(-)}(E)\right\rangle \\
& \times\left\langle\chi_{\mathcal{E}}^{(-)}(E)\left|\sqrt{1-\mathcal{V}^{\prime}(E)} \hat{j}^{\mu}(\boldsymbol{q})\right| \varphi_{n}\right\rangle .
\end{aligned}
$$

The factor $\sqrt{1-\mathcal{V}^{\prime}(E)}$, where $\mathcal{V}^{\prime}(E)$ is the energy derivative of the optical potential, accounts for interference effects between different channels and justifies the replacement in the calculations of the Feshbach optical potential $\mathcal{V}$, for which neither microscopic nor empirical calculations are available, by the local phenomenological optical potential $[12,13]$. Disregarding the square root correction, the second matrix element in Eq. (3) is the transition amplitude of single-nucleon knockout of Eq. (1), where the imaginary part of the optical potential accounts for the flux lost in the channel $n$ towards the channels different from $n$. In the inclusive response this loss is compensated by a corresponding gain of flux due to the flux lost, towards the channel $n$, by the other final states asymptotically originated by the channels different from $n$. This compensation is performed by the first matrix element in the right 
hand side of Eq. (3), that is similar to the matrix element of Eq. (1) but involves the eigenfunction $\tilde{\chi}_{\mathcal{E}}^{(-)}(E)$ of the Hermitian conjugate optical potential, where the imaginary part has an opposite sign and has the effect of increasing the strength. As a consequence, in the GF model the imaginary part of the optical potential redistributes the flux lost in a channel in the other channels, and in the sum over $n$ the total flux is conserved.

The hadron tensor in Eq. (2) is the sum of two terms. The calculation of the second term requires the integration over all the eigenfunctions of the continuum spectrum of the optical potential. If the imaginary part of the optical potential is neglected, the second term in Eq. (2) vanishes and, but for the square root factor, the first term gives the rROP approach.

In the usual applications of the GF model the matrix elements in Eq. (2) are calculated using the same phenomenological bound and scattering states already adopted in DWIA and RDWIA calculations for exclusive one-nucleon knockout reactions. For the sum over $n$ a pure shell model description is usually assumed: $\varphi_{n}$ are one-hole states in the target nucleus with a unitary spectra strength and the sum is over all the occupied states in the shell model. With this simplifying assumption the contribution of all the nucleons is correctly included in the inclusive response.

The GF model allows to recover the contribution of non-elastic channels starting from the complex optical potential that describes elastic nucleon-nucleus scattering data. It provides a consistent treatment of FSI in the exclusive and in the inclusive scattering and gives also a good description of $\left(e, e^{\prime}\right)$ data $[12,13,19]$.

Both nonrelativistic GF $[12,16]$ and relativistic RGF [13] models have been considered for the inclusive electron scattering, while only the RGF has been used for CC neutrino scattering [14]. The results of the RMF and RGF models have been compared in [19] for the inclusive electron scattering and in [20] for the inclusive CC neutrino scattering.

An example is displayed in figure 1, where the RGF, $\mathrm{RMF}$, rROP, and RPWIA cross sections of the ${ }^{12} \mathrm{C}\left(e, e^{\prime}\right)$ reaction calculated in a kinematics with a fixed value of the incident electron energy $(\varepsilon=1 \mathrm{GeV})$ and two values of the momentum transfer $(q=500$ and $1000 \mathrm{MeV} / c)$ are compared. Two parameterizations of the ROP have been used for the RGF calculations: the energy-dependent and A-dependent EDAD1 (RGF-EDAD1) and EDAD2 (RGFEDAD2) [21].

The RGF and RMF results are always different from the results of the simpler RPWIA and rROP approaches. The differences between RMF and RGF, as well as the differences between RGF-EDAD1 and RGF-EDAD2, increase with the momentum transfer. At $q=500 \mathrm{MeV} / c$ the three results are similar, both in magnitude and shape, at $q=1000 \mathrm{MeV} / c$, the shape of the RMF cross section shows an asymmetry, with a tail extending towards higher values of the energy transfer $\omega$, which is essentially due to the strong energy-independent scalar and vector potentials present in the RMF model. The asymmetry towards higher $\omega$ is less significant but still visible for RGF-EDAD1 and RGF-EDAD2, whose cross sections show a similar shape but a significant difference in magnitude. At momentum transfer $q=1000 \mathrm{MeV} / c$ both the RGF-EDAD1 and RGFEDAD2 cross sections are higher than the RMF one in the
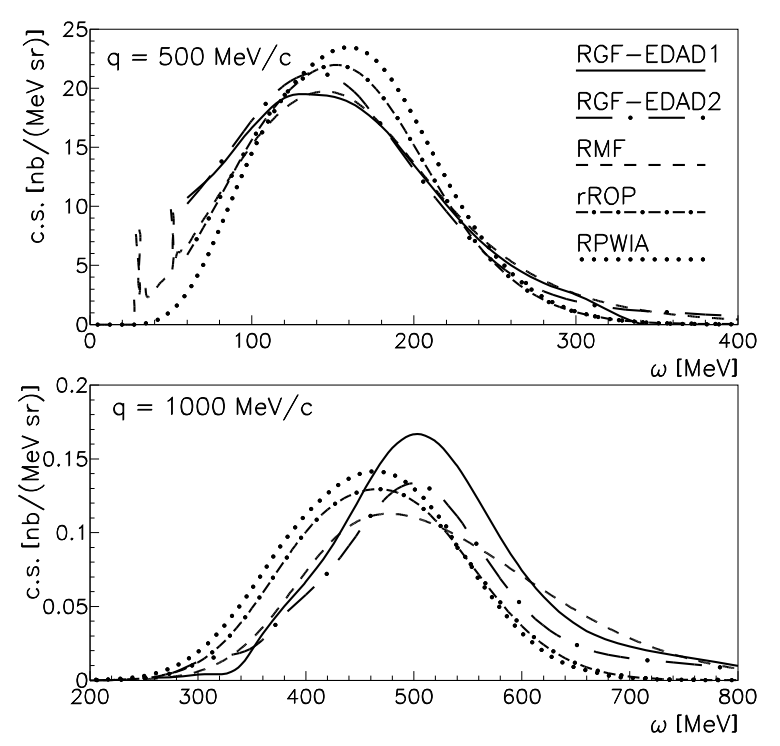

Fig. 1. Differential cross section of the ${ }^{12} \mathrm{C}\left(e, e^{\prime}\right)$ reaction for an incident electron energy $\varepsilon=1 \mathrm{GeV}$ and $q=500$ and 1000 $\mathrm{MeV} / c$ as a function of the energy transfer $\omega$. Results for RPWIA (dotted), rROP (dot-dashed), RGF-EDAD1 (solid), RGF-EDAD2 (long dot-dashed), and RMF (dashed) are compared.

maximum region, but a stronger enhancement is obtained with RGF-EDAD1, which at the peak overshoots the RMF cross section up to $40 \%$ and it is even higher than the RPWIA result.

The differences between the RGF-EDAD1 and RGFEDAD2 results are basically due to the differences in the imaginary part of the two optical potentials. The real terms are very similar for different parameterizations and the rROP cross sections are not sensitive to the parameterization considered.

In figure 2 the RGF, RMF, rROP, and RPWIA results are compared for the cross sections of the ${ }^{12} \mathrm{C}\left(v_{\mu}, \mu^{-}\right)$reaction calculated with the same incident lepton energy and momentum transfer as for the $\left(e, e^{\prime}\right)$ reaction of figure 1 . For the RGF model, the RGF-EDAD1 results are compared with the results obtained with the energy-dependent but A-independent EDAI potential (RGF-EDAI). Also in figure 2 the shape of the RMF cross section shows an asymmetry with a tail extending towards higher values of $\omega$ (corresponding to lower values of the kinetic energy of the outgoing muon $T_{\mu}$ ). An asymmetric shape is shown also by the RGF cross sections, while no visible asymmetry is given by the RPWIA and rROP results. Also in this case the differences between the two RGF results are due to the different imaginary parts of the two ROP's. As already shown for the $\left(e, e^{\prime}\right)$ reaction, the RGF yields in general a larger cross section than the RMF.

The results in figure 2 present some differences with respect to the corresponding $\left(e, e^{\prime}\right)$ cross sections in figure 1 . In both cases the differences between the results of the different models are generally larger for increasing value of the momentum transfer. For neutrino scattering, however, such a behavior is less evident and clear. In particular, the RGF-EDAD1 cross section at $q=1000 \mathrm{Mev} / c$ does not show the strong enhancement in the region of the maximum shown in figure 1, where the RGF-EDAD1 result is 


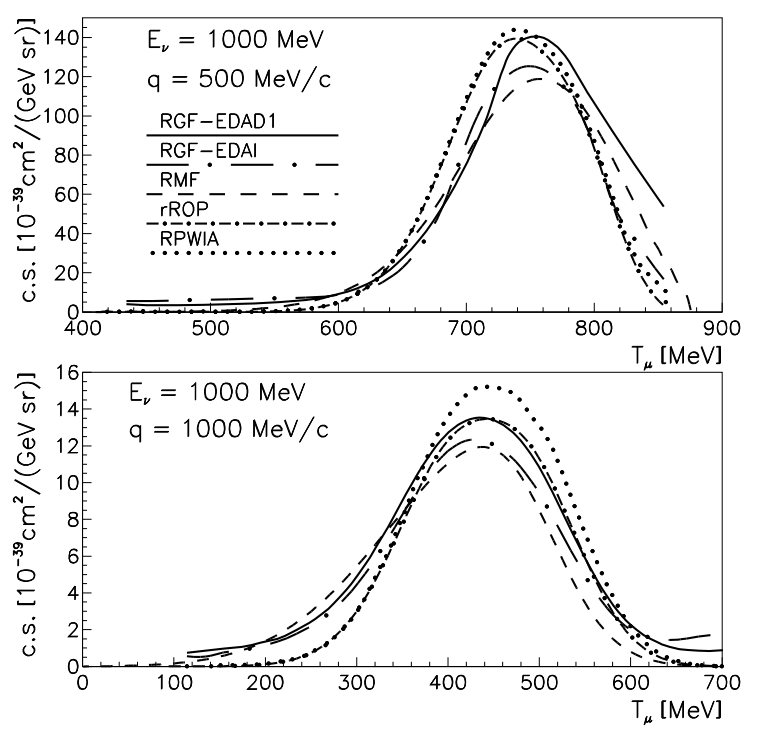

Fig. 2. Differential cross section of the ${ }^{12} \mathrm{C}\left(v_{\mu}, \mu^{-}\right)$reaction for $\mathrm{E}_{v}$ $=1000 \mathrm{MeV}$ and $q=500 \mathrm{MeV} / c$ and $1000 \mathrm{MeV} / c$ as a function of the kinetic energy of the outgoing muon $T_{\mu}$. Results for RPWIA (dotted), rROP (dot-dashed) RGF-EDAD1 (solid), RGF- EDAI (long dot-dashed), and RMF (dashed) are compared.

even larger than the RPWIA one. In the case of neutrino scattering the RGF results in the region of the maximum are generally larger than the RMF ones, but smaller than the RPWIA cross sections. The numerical differences between the RGF results for electron and neutrino scattering can mainly be ascribed to the combined effects of the weak current, in particular its axial term, and the imaginary part of the ROP [20].

We recall that the RMF model uses as input the real, strong, energy-independent, relativistic mean field potential that reproduces the saturation properties of nuclear matter and of the ground state of the nuclei involved. As such, it includes only purely nucleonic contribution and does not incorporate any information from scattering reactions. In contrast, the RGF uses as input the complex energydependent relativistic optical potential. Phenomenological optical potentials, obtained through a fit of elastic protonnucleus scattering data, are adopted in actual RGF calculations. Therefore, the RGF model incorporates information from scattering reactions and takes into account not only direct one-nucleon emission, but all the allowed final states, as the s.p. Green's function contains the full propagator.

The imaginary part of the ROP includes the overall effect of all the inelastic channels, which give different contributions at different energies. This energy dependence makes the RGF results strongly dependent on kinematics. The differences between the RGF and RMF results can be ascribed to the inelastic contributions which are incorporated in the RGF but not in the RMF (and in other models based on the IA), such as, for instance, re-scattering processes of the nucleon in its way out of the nucleus, non-nucleonic $\Delta$ excitations, which may arise during nucleon propagation, as well as to some multinucleon processes. These contributions are not included explicitly in the RGF model with a microscopic approach, but they can be recovered, to some extent, by the imaginary part of the phenomenological optical potential. With the use of such a phenomenological ingredient, however, we cannot disentangle the role of different reaction processes and explain in detail the origin of the differences, but we can expect that the differences increase with the relevance of such inelastic contributions.

The comparison between the RGF and RMF results can therefore be useful to evaluate the relevance of inelastic contributions. If in many situations the two models give close predictions (usually different from those of the simpler RPWIA and rROP), there are also situations where the differences are large $[19,20]$.

In the comparison with data, we may expect that the RGF can give a better description of those experimental cross sections which receive significant contributions from non-nucleonic excitations and multi-nucleon processes. This is expected to be the case [22,23] of MiniBooNE data, given the nature of the experiment. While in electron-scattering experiments the beam energy is known and the cross sections are given as a function of the energy transfer, in neutrino experiments $q$ and $\omega$ are not known and calculations for the comparison with data are carried out over the energy range which is relevant for the neutrino flux. The flux-average procedure can include contributions from different kinematic regions where the neutrino flux has significant strength and processes other than direct one-nucleon emission can be important $[24,25]$. Part of these contributions are recovered in the RGF model by the imaginary part of the optical potential

\section{Comparison with CCQE MiniBooNE data}

The CCQE ${ }^{12} \mathrm{C}\left(v_{\mu}, \mu^{-}\right)$cross sections recently measured by the MiniBooNE collaboration [1] have raised debate over the role of the theoretical ingredients entering the description of the reaction. The experimental cross section is underestimated by the Relativistic Fermi Gas (RFG) model, and by other more sophisticated models based on the IA, unless the nucleon axial mass $M_{A}$ is significantly enlarged $\left(1.35 \mathrm{GeV} / c^{2}\right.$ in the RFG) with respect to the accepted world average of all measured values $\left(1.03 \mathrm{GeV} / c^{2}[26\right.$, 27]), mostly obtained from deuteron data. The larger axial mass obtained from the MiniBooNE data on carbon can also be interpreted as an effective way to include medium effects which are not taken into account by the RFG and by other models. Before drawing conclusions, it is therefore important to evaluate carefully the role played by all the nuclear effects.

The effect of FSI is investigated in figure 3, where the CCQE double-differential ${ }^{12} \mathrm{C}\left(v_{\mu}, \mu^{-}\right)$cross sections averaged over the neutrino flux are displayed as a function of $T_{\mu}$ for various bins of $\cos \theta$, where $\theta$ is the muon scattering angle. The RMF results yield reasonable agreement with data for small angles and low muon energies, the discrepancy becoming larger as $\theta$ and $T_{\mu}$ increase. The shape followed by the RMF and RGF cross sections fits well the slope shown by the data. The two models yield close predictions at larger values of $T_{\mu}$, for all the bins of $\cos \theta$ shown in the figure. The RGF cross sections are generally larger than the RMF ones. The differences increase approaching the peak region, where the additional strength shown by the RGF produces cross sections in reasonable 

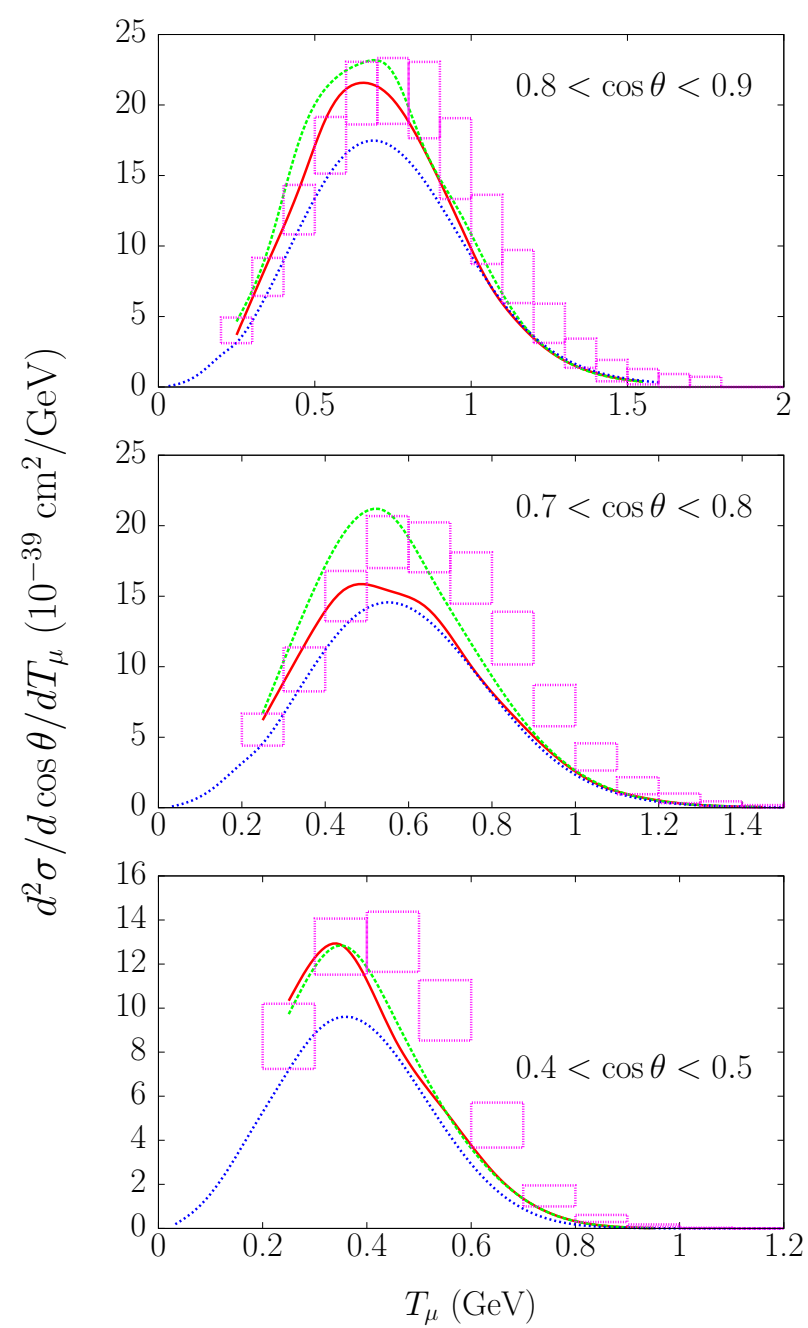

Fig. 3. Flux-averaged double differential cross section per target nucleon for the $\mathrm{CCQE}{ }^{12} \mathrm{C}\left(v_{\mu}, \mu^{-}\right)$reaction calculated in the RMF (blue line) and in the RGF with EDAD1 (red) and EDAI (green) potentials and displayed versus $T_{\mu}$ for various bins of $\cos \theta$. The data are from MiniBooNE [1]. The uncertainties do not include the overall normalization error $\delta N=10.7 \%$.

agreement with the data. The differences between the RGFEDAI and RGF-EDAD1 results are enhanced in the peak region and are in general of the order of the experimental errors.

In figure 4 the total CCQE cross sections per neutron obtained in the RMF, RGF, rROP, and RPWIA models are displayed as a function of the neutrino energy and compared with the "unfolded" experimental data [1]. The rROP, RPWIA, and RMF results usually underpredict the MiniBoone cross section. It is shown in $[28,29]$ that the differences between models like the RMF, rROP, RPWIA, and superscaling tend to be washed out in the integration and that all these models, which represent essentially the same nucleonic contribution to the inclusive cross sections, undepredict the total MiniBooNE CCQE cross section, whereas the inclusion of two-particle-two-hole meson-exchange currents enhances the results.

Larger cross sections, in particular for larger values of $E_{v}$, are obtained in the RGF with both optical potentials. The differences between the RGF-EDAI and the RGF-EDAD1 results, being RGF-EDAI in good agreement with the shape and magnitude of the experimental cross section

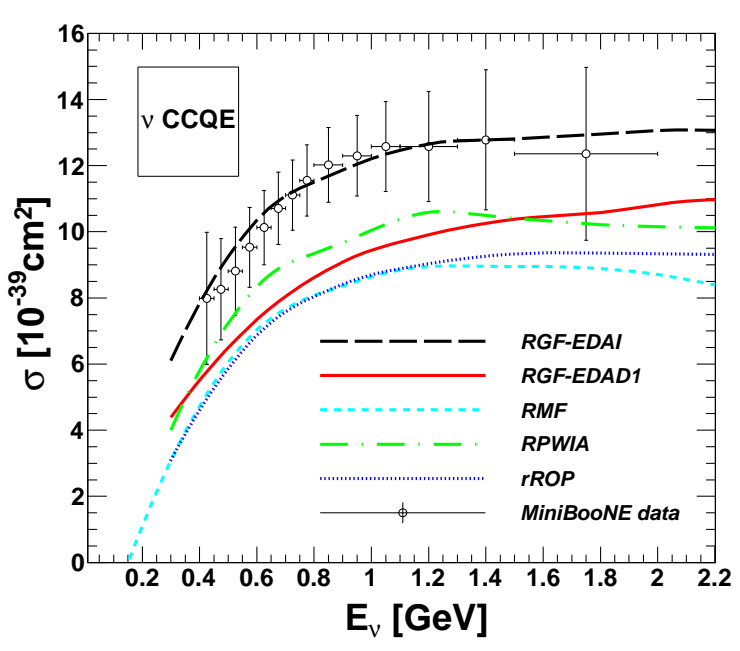

Fig. 4. Total CCQE cross section per target nucleon as a function of the neutrino energy $E_{v}$ calculated with the RGF-EDAD1 (solid line), the RGF-EDAI (long-dashed line), the RMF (dashed line), the rROP (dotted line), and the RPWIA (dot-dashed line). The experimental data for neutrino scattering are from MiniBooNE [1].

and RGF-EDAD1 above RMF but clearly below the data, are due to the different imaginary parts of the two ROP's, particularly for the energies considered in kinematics with the lowest $\theta$ and the largest $T_{\mu}$. We notice that EDAI is a single-nucleus parameterization, which does have an edge in terms of better reproduction of the elastic proton- $-{ }^{12} C$ phenomenology [21] compared to EDAD1, and also leads to CCQE results in better agreement with data.

The MiniBooNE collaboration has also measured $\bar{v}_{\mu}$ CCQE events. The data analysis is currently ongoing [30] and some preliminary results can be found on the MiniBooNE website [31]. When available, the antineutrino measurements will be an additional source of information about the weak charged-current lepton-nucleus interaction.

In figure 5 the total CCQE cross sections per target nucleon calculated with the RGF, RPWIA, and rROP for antineutrino scattering are displayed as a function of the antineutrino energy $E_{\bar{v}}$. Also for $\bar{v}$ scattering the RGF results are usually larger than the RPWIA and rROP ones. The differences between the RGF-EDA1 and RGF-EDAI results are significant also for antineutrino scattering, although somewhat smaller than for neutrino scattering. Moreover, we note that the antineutrino cross section does not saturate in the energy range up to $\approx 2 \mathrm{GeV}$ which we have considered. The different behavior of the cross sections calculated for neutrino and antineutrino scattering is related to the relative strength of the vector-axial response, which is constructive in $v$ scattering and destructive in $\bar{v}$ scattering with respect to the longitudinal and transverse ones [32].

The MiniBooNE Collaboration has recently reported [2] also a measurement of the flux-averaged differential cross section as a function of the four-momentum transferred squared, $Q^{2}=-q^{\mu} q_{\mu}$, for neutral-current elastic (NCE) neutrino scattering on $\mathrm{CH}_{2}$ in a $Q^{2}$ range up to $\approx 1.65(\mathrm{GeV} / c)^{2}$. A careful analysis of $v$-nucleus NCE reactions introduces additional complications, as the final neutrino cannot be measured in practice and a final hadron 


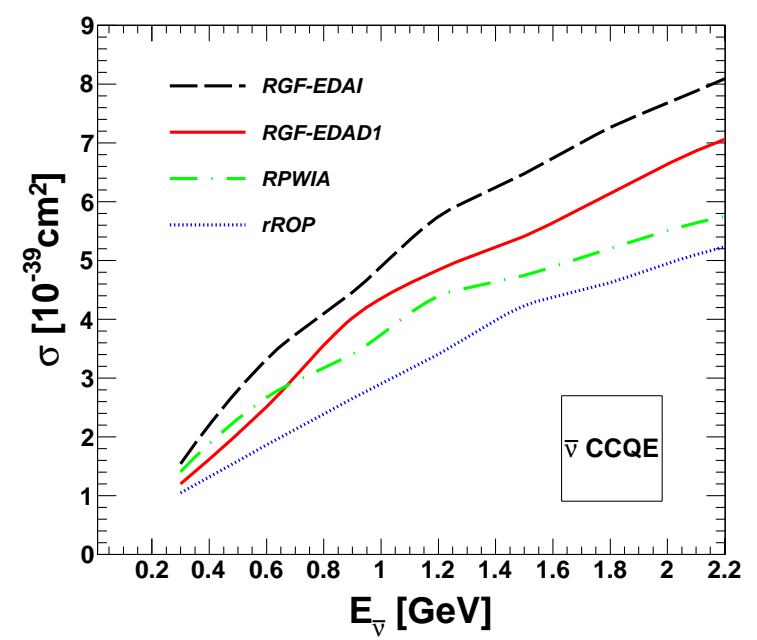

Fig. 5. Total CCQE cross section per target nucleon as a function of the antineutrino energy $E_{\bar{v}}$ calculated with the RGF-EDAD1 (solid line), the RGF-EDAI (dashed line), the rROP (dotted lines), and the RPWIA (dot-dashed line).

has to be detected: the corresponding cross sections are therefore semi-inclusive in the hadronic sector and inclusive in the leptonic one. Different relativistic descriptions of FSI are presented and compared with the NCE MiniBooNE data in [33].

\section{Summary and conclusions}

A deep understanding of the reaction mechanism of neutrino-nucleus cross sections is mandatory for the determination of neutrino oscillation parameters. Reliable theoretical models are required where all nuclear effects are well under control. Within the QE kinematics domain, the treatment of FSI is an essential ingredient for the comparison with data.

Models developed for QE electron scattering and successfully tested in comparison with electron-scattering data have been extended to neutrino-nucleus scattering. The results of different relativistic models for the inclusive QE electron and neutrino-nucleus scattering, with different descriptions of FSI, have been compared in this contribution. In the relativistic plane-wave impulse approximation (RPWIA) FSI are simply neglected. In other approaches FSI are included in RDWIA calculations where the final nucleon state is evaluated with real potentials, either retaining only the real part of the relativistic energy-dependent complex optical potential (rROP), or using the same relativistic mean field potential considered in describing the initial nucleon state (RMF). In the relativistic Green's function (RGF) model FSI are described in the inclusive scattering by the same complex optical potential as in the exclusive scattering, but the imaginary part is used in the two cases in a different way and in the inclusive process it is responsible for the redistribution of the flux in all the channels and the total flux is conserved.

The differences between the results of the different models depend on kinematics. The predictions of the RGF and RMF models are close in many situations, and generally different from the RPWIA and rROP results. There are, however, also situations where the differences are large.
Larger cross section are generally obtained with the RGF model, which is able to give a better description of experimental data. In particular, the RGF model is able to reproduce the CCQE MiniBoone cross sections without the need to increase the standard value of the nucleon axial mass. The enhancement of the RGF cross sections is due to the translation to the inclusive strength of the overall effect of inelastic channels, due, for instance, to re-scattering processes, non-nucleonic excitations, which may arise during nucleon propagation, or to some multinucleon processes. Such inelastic contribution, which are not incorporated in the RMF and in other models based on the impulse approximation, are not included explicitly in the RGF model with a microscopic approach, but they can be recovered, to some extent, by the imaginary part of the optical potential. The use of phenomenological optical potentials, however, does not allow us to disentangle the role of different reaction processes and explain in detail the origin of the enhancement.

Other models, where multinucleon components are explicitly included, are also able to describe the MiniBooNE data without increasing the value of the axial mass [34 38]. The important role of contributions other than direct one-nucleon emission is therefore confirmed by different and somewhat alternative models. A careful and consistent evaluation of all nuclear effects is required before definite conclusions can be drawn. A detailed comparison of the models and of their results would be helpful for a deeper understanding.

Processes involving two-body currents, whose role has been discussed in [25,27-29], should also be taken into account explicitly and consistently in a model to clarify the role of multinucleon emission. Fully relativistic microscopic calculations of two-particle-two-hole (2p-2h) contributions are extremely difficult and may be bound to model-independent assumptions.

The RGF results are also affected by uncertainties in the determination of the phenomenological optical potential. At present, lacking a phenomenological optical potential which exactly fulfills the dispersion relations in the whole energy region of interest, the RGF prediction is not univocally determined from the elastic phenomenology. The differences of the results obtained with different parameterizations of the relativistic optical potential are produced by the different imaginary part, which is the crucial ingredient of RGF calculations. It is interesting to notice that the best predictions in comparison with data are given by the EDAI potential, that is also able to give the best description of the elastic proton- ${ }^{12} \mathrm{C}$ phenomenology. A better determination of a phenomenological relativistic optical potential, which closely fulfills the dispersion relations, would be anyhow desirable and deserves further investigation.

\section{Acknowledgements}

We thank F.D. Pacati, F. Capuzzi, J.A. Caballero, J.M. Udías, and M.B. Barbaro for the fruitful collaborations that led to the results reported in this contribution. This work has been partially supported by the Italian MIUR through the PRIN 2009 research project. 


\section{References}

1. A.A. Aguilar-Arevalo, et al. (MiniBooNE Collaboration), Phys. Rev. D 81, 092005 (2010)

2. A.A. Aguilar-Arevalo, et al. (MiniBooNE Collaboration), Phys. Rev. D 82, 092005 (2010)

3. S. Boffi, C. Giusti, and F.D. Pacati, Phys. Rep. 226, 1 (1993)

4. S. Boffi, C. Giusti, F.D. Pacati, and M. Radici Electromagnetic Response of Atomic Nuclei (Oxford Studies in Nuclear Physics 20, Clarendon Press, Oxford 1996)

5. S. Boffi et al., Nucl. Phys. A 379, 509 (1982)

6. A. Meucci, C. Giusti, and F.D. Pacati, Phys. Rev. C 64, 014604 (2001)

7. J.M. Udías, et al., Phys. Rev. C 48, 2731 (1993)

8. M. Radici, A. Meucci, and W.H. Dickhoff, Eur. Phys. J. A 17, 65 (2003)

9. C. Maieron, et al., Phys. Rev. C 68, 048501 (2003)

10. J.A. Caballero, et al., Phys. Rev. C 74, 015502 (2006)

11. Y. Horikawa, F. Lenz, and N.C. Mukhopadhyay, Phys. Rev. C 22, 1680 (1980)

12. F. Capuzzi, C. Giusti, and F.D. Pacati, Nucl. Phys.A 524, 681 (1991)

13. A. Meucci, F. Capuzzi, C. Giusti, and F.D. Pacati, Phys. Rev. C 67, 054601 (2003)

14. A. Meucci, C. Giusti, and F.D. Pacati, Nucl. Phys. A 739, 277 (2004)

15. A. Meucci, C. Giusti, and F.D. Pacati, Nucl. Phys. A 756, 359 (2005)

16. F. Capuzzi, C. Giusti, F.D. Pacati, and D.N. Kadrev, Ann. Phys. 317, 492 (2005)

17. A. Meucci, C. Giusti, and F.D. Pacati, Acta Phys. Polon. B 37, 2279 (2006)

18. A. Meucci, C. Giusti, and F.D. Pacati, Acta Phys. Polon. B 40, 2579 (2009)

19. A. Meucci, et al., Phys. Rev. C 80, 024605 (2009)

20. A, Meucci, J.A. Caballero, C. Giusti, and J.M. Udías, Phys. Rev. C 83, 064614 (2011)

21. E.D. Cooper, et al., Phys. Rev. C 47, 297 (1993)

22. T. Leitner and U. Mosel, Phys. Rev. C 81, 064614 (2010)

23. A. Meucci, et al., Phys. Rev. Lett. 107, 172501 (2011)

24. O. Benhar, P. Coletti, and D. Meloni, Phys. Rev. Lett. 105, 132301 (2010)

25. O. Benhar and G. Veneziano, Phys. Lett. B 702, 433 (2011)

26. V. Bernard, L. Elouadrhiri, and U.G. Meissner, J. Phys. G: Nucl. Part. Phys. 28, R1 (2002)

27. A. Bodek, et al., Eur. Phys. J. C 53, 349 (2008)

28. J.E. Amaro, et al., Phys. Rev. D 84, 033004 (2011)

29. J.E. Amaro, et al., Phys. Rev. Lett. 108, 152501 (2012)

30. A.A. Aguilar-Arevalo, et al. (MiniBooNE Collaboration), Phys. Rev. D 84, 072005 (2011)

31. http://www-boone.fnal.gov

32. A. Meucci and C. Giusti, Phys. Rev. D 85, 093002 (2012)

33. A. Meucci, C. Giusti, and F.D. Pacati, Phys. Rev. D 84, 113003 (2011)

34. M. Martini, M. Ericson, G. Chanfray, and J. Marteau, Phys. Rev. C 80, 065501 (2009)

35. M. Martini, M. Ericson, G. Chanfray, and J. Marteau, Phys. Rev. C 81, 045502 (2010)

36. M. Martini, M. Ericson, and G. Chanfray, Phys. Rev. C 84, 055502 (2011)
37. J. Nieves, I. Ruiz Simo, and M.J. Vicente Vacas, Phys. Rev. C 83, 045501 (2011)

38. J. Nieves, I. Ruiz Simo, and M.J. Vicente Vacas, Phys. Lett. B 707, 72 (2012) 